\title{
Uso de álcool, conflitos familiares e supervisão parental entre estudantes do ensino médio
}

\author{
Alcohol use, family conflicts and parental supervision \\ among high school students
}

João Victor Viana da Silva Neves (https://orcid.org/0000-0003-1254-6425) ${ }^{1}$

Lucas Almeida de Carvalho (https://orcid.org/0000-0001-6982-9878) ${ }^{1}$

Mateus Almeida de Carvalho (https://orcid.org/0000-0001-5836-6468) ${ }^{1}$

Érica Thaís Costa Silva (https://orcid.org/0000-0002-9785-9844) ${ }^{1}$

Maria Luiza Terra Santos Alves (https://orcid.org/0000-0002-4600-0562) ${ }^{2}$

Marise Fagundes Silveira (https://orcid.org/0000-0002-8821-3160) ${ }^{2}$

Rosângela Ramos Veloso Silva (https://orcid.org/0000-0003-3329-8133) ${ }^{2}$

Maria Tereza Carvalho Almeida (https://orcid.org/0000-0003-2060-2239) ${ }^{2}$
${ }^{1}$ Centro Universitário FIPMoc. Av. Profa. Aida Mainartina Paraiso 80, Ibituruna. 39408-007 Montes Claros MG Brasil. jvsneves27@yahoo.com ${ }^{2}$ Universidade Estadual de Montes Claros. Montes Claros MG Brasil.

\begin{abstract}
The association between alcohol consumption by students with family conflicts, parental supervision and quality of relationships was investigated. A cross-sectional study was conducted with 1,265 adolescents from 21 schools. The Brazilian version of the Drug Use Screening Inventory/DUSI was used, and absolute and relative prevalence for categorical variables was estimated, while bivariate analyses and Pearson's Chi Square test were conducted. For the magnitude of the association between the dependent and independent variables, prevalence ratios were estimated using Poisson multivariate regression. There was an association between alcohol consumption and drug use by family members, frequent discussions and parental lack of awareness about adolescents' activities. Family supervision, a good relationship with parents/guardians, establishing an affective family bond and parents' knowledge about teenagers' whereabouts, were protective factors for alcohol use. The conclusion was that the high rate of schoolchildren who consume alcohol is worrying, liable to health complications in general, and a harmonized family relationship is fundamental. The implementation of measures of intervention between school and the Family Health Strategy, can contribute to the establishment of a protective bond.
\end{abstract}

Key words Adolescent, Alcoholic beverages, $\mathrm{Fa}$ mily conflict, Family relationships
Resumo Investigou-se a associação entre o consumo de álcool pelos escolares com os conflitos familiares, supervisão dos pais e qualidade dos relacionamentos. Estudo transversal realizado com 1.265 adolescentes de 21 escolas. Foi utilizada a versão brasileira do Inventário de Triagem do Uso de Drogas/DUSI e estimadas prevalências absolutas e relativas para variáveis categóricas, $e$ conduzidas análises bivariadas: teste Qui Quadrado de Pearson. Para magnitude da associação entre a variável dependente e independentes foram estimadas razões de prevalências por meio deregressão multivariável de Poisson. Houve associação do consumo de álcool com uso de drogas por familiares, discussões frequentes e desconhecimento dos pais sobre o que os adolescentes fazem. Supervisão familiar, bom relacionamento com pais/responsáveis, estabelecimento de vínculo familiar afetivo e ciência dos pais acerca do que os adolescentes fazem e onde estão, foram fatores de proteção para uso de álcool. Concluiu-se que a alta taxa de escolares que consomem álcool é preocupante, passível de complicações da saúde na sua integralidade, sendo fundamental relação familiar harmonizada. A implementação de práticas interventivas entre escola e Estratégia de Saúde da Família, podem contribuir no estabelecimento de vinculo protetivo.

Palavras-chave Adolescente, Bebidas alcoólicas, Conflito familiar, Relações familiares 


\section{Introdução}

A adolescência, fase compreendida entre 10 e 19 anos $^{1}$, é o momento em que há a construção de sistemas e teorias, e no sujeito a necessidade de se integrar em um mundo diferente buscando identificações exteriores ${ }^{2}$. Constitui um período de dúvidas, conflitos, mudanças, descobertas ${ }^{3} \mathrm{e}$ de transformações significativas no corpo, o que implica em alterações na personalidade e na forma de lidar com o outro e com a sociedade ${ }^{4}$, em especial com a família, uma instituição que exerce influência significativa durante todo o processo de desenvolvimento do indivíduo 5 .

Drogas, condições socioeconômicas e preocupações com o corpo são temáticas ressaltadas na fala dos adolescentes, delineiam os contornos socioculturais e os expõe a confrontar-se com os limites e possibilidades de sua existência ${ }^{6}$. O uso de álcool é uma questão complexa que abrange dimensões individual, familiar, social, política e econômica ${ }^{7}$ e tem se destacado como um dos principais fatores de risco para a saúde, podendo comprometer o desenvolvimento do cérebro, incluindo aspectos cognitivo, emocional e social ${ }^{8}$. Pesquisa nacional de saúde do escolar mostrou que mais de $70 \%$ dos adolescentes haviam experimentado bebidas alcoólicas e $26,1 \%$ haviam consumido álcool nos últimos 30 dias $^{8}$.

Bock et al. ${ }^{9}$ destacam a importância do papel da família no desenvolvimento psicossocial/afetivo do indivíduo, para estes a família reproduz no ambiente a cultura e seus costumes que posteriormente o sujeito internalizará. É na família que se desenvolve as bases estruturais psíquicas do ser humano construindo uma identidade individual e social, tornando-se mais aptos a lidar com as dificuldades ${ }^{10}$. Entretanto, os adolescentes têm forte tendência a se distanciarem da família tornando a comunicação cada vez mais difícil, favorecendo desentendimentos e distanciamento no convívio familiar ${ }^{11}$.

Portanto, ao experimentaremtodas as mudanças nessa fase da vida, os adolescentes vivenciam a exposição a diversos fatores de risco comportamentais, como o consumo de álcool, tornando-se mais vulneráveis ${ }^{8}$. Assim, estudos que proporcionem conhecimento mais amplo sobre tal problemática no intuito de fornecer intervenções eficazes de prevenção e de controle do uso de álcool entre adolescentes tornam-se necessários?.

Este estudo teve como objetivo investigar a associação do uso de álcoolpelos adolescentes inseridos no contexto escolar com os conflitos familiares, supervisão dos pais e qualidade dos relacionamentos.

\section{Métodos}

Este é um recorte de um estudo que investigou o uso de drogas entre adolescentes da rede pública de ensino da cidade de Montes Claros, Minas Gerais, Brasil. Trata-se de um estudo transversal do tipo epidemiológico de base institucional (escolas), realizado com estudantes do ensino médio da zona urbana.

O município está localizado no norte do estado de Minas Gerais, na área do "Polígono das Secas" e caracteriza-se por seus aspectos fisiográficos (zona de transição cerrado/caatinga) e pelos seus baixos indicadores socioeconômicos. Montes Claros assume a posição de centralidade nessa região, constituindo o núcleo urbano mais expressivo, desempenhando a função de centralizar o comércio varejista, interrelação política e administrativa, serviços de saúde, ensino de nível superior, entre outros. Com uma população estimada em 410.000 habitantes, possui 38 unidades de escolas públicas da rede estadual com ensino médio, a partir de listagem estratificada oferecida pela Secretaria de Estado de Educação em Setembro de 2016, com 13.104 escolares matriculados no ano de 2017.

A amostra foi definida por meio de cálculo amostral para populações finitas, considerando a prevalência do evento de interesse em $50 \%$, nível de confiança de $95 \%$, margem de erro de $5 \%$, Deff $=2$ e acréscimo de $10 \%$ para compensar possíveis perdas. A seleção da amostra foi do tipo probabilística por conglomerados em dois estágios, sendo o primeiro constituído pelas escolas e o segundo pelas turmas das escolas selecionadas. No primeiro estágio, as escolas foram selecionadas por amostragem probabilística proporcional ao tamanho (PPT). No segundo estágio, foi definida por amostragem aleatória simples, foi selecionada uma fração amostral das turmas em cada uma das escolas sorteadas, estratificadas por turno (matutino, vespertino e noturno). A fração amostral foi definida após o sorteio das escolas.

O tamanho mínimo calculado foi de 1.768 adolescentes. Considerando esses parâmetros para garantir representatividade da amostra, verificou-se a necessidade de incluir alunos distribuídos em 21 escolas, sendo aplicados questionários em 2.040 escolares. A amostra foi portanto superior ao mínimo exigido no cálculo amostral. No entanto, seguindo os parâmetros da "Escala 
de Mentiras" do questionário DUSI, 775 questionários foram excluídos, pois os adolescentes marcaram incorretamente a última questão do bloco de investigação. Assim, este estudo considerou 1.265 adolescentes participantes. Os dados foram coletados por uma equipe multiprofissional e por estudantes de iniciação científica, entre maio de 2017 e março de 2018, em dias agendados previamente em cada escola selecionada.

Todos os alunos selecionados por sorteio foram convidados a participar. Foram incluídos alunos de ambos os sexos, idade entre $14 \mathrm{e}$ 19 anos, devidamente matriculados. Foram excluídos da pesquisa os alunos que não estavam em sala de aula no momento da aplicação do questionário, aqueles que não estavam em concordância com o objetivo da pesquisa e os que não apresentaram TALE (Termo de Assentimento Livre e Esclarecido) e TCLE (Termo de Consentimento Livre e Esclarecido) devidamente assinados. Para a coleta de dados, utilizou-se um questionário autoaplicável. Após esclarecimentos sobre a pesquisa e entregue a autorização devidamente assinada pelos pais e/ou responsáveis, os alunos foram encaminhados para uma sala, onde foram aplicados os questionários. O questionário autoaplicado foi preenchido por cada adolescente individualmente. Durante o preenchimento do questionário, membros da equipe do projeto estavam disponíveis para auxiliar e esclarecer dúvidas, caso necessário.

O questionário avaliou características sociodemográficas e a versão brasileira do Inventário de Triagem do Uso de Drogas (DUSI) adaptado e validado para o Brasil ${ }^{12}$, sendo abordada a área VI do DUSI que investiga os conflitos familiares, supervisão dos pais e qualidade de relacionamento. No questionário sociodemográfico, foram obtidas informações referentes ao ano de escolaridade, horário de aula, sexo, idade, cor da pele, renda, classe econômica e uso de álcool. As variáveis da área VI do DUSI foram: "Algum membro de sua família (mãe, pai, irmão ou irmã) usou maconha ou cocaína no último ano?"; "Algum membro de sua família usou álcool a ponto de causar problemas em casa, no trabalho ou com amigos?"; "Algum membro de sua família foi preso no último ano?"; "Você tem tido discussões frequentes com seus pais ou responsáveis que envolvam gritos e berros?"; "Sua família dificilmente faz coisas juntas?"; "Seus pais ou responsáveis desconhecem o que você gosta e o que não gosta?"; "Na sua casa faltam regras claras sobre o que você pode e não pode fazer?; Seus pais ou responsáveis brigam muito entre si?"; "Seus pais ou responsáveis fre- quentemente desconhecem onde você está ou o que você está fazendo?"; "Seus pais ou responsáveis estão fora de casa a maior parte do tempo?"; "Você sente que seus pais ou responsáveis não se importam ou não cuidam de você?"; "Você se sente infeliz em relação ao local no qual você vive?"; e "Você se sente em perigo em casa?".

Os dados foram digitados, tabulados, auditados e analisados com auxílio do programa Statistical Package for Social Sciences (SPSS ${ }^{\circledR}$ ), versão 22.0. Na condução das análises descritivas, foram estimadas prevalências absolutas e relativas para as variáveis categóricas. Sequencialmente, foram conduzidas análises bivariadas através do Teste Qui Quadrado de Pearson. Nesta análise, todas as variáveis que revelaram nível descritivo inferior a $20 \%(p<0,2)$ foram selecionadas para compor inicialmente a regressão multivariável de Poisson. Os modelos foram ajustados mantendo-se ao final apenas as variáveis com nível descritivo inferior a $5 \%(\mathrm{p}<0,05)$. Para avaliar a magnitude da associação entre a variável dependente e as variáveis independentes foi estimada razão de prevalência (RP), com intervalo de $95 \%$ de confiança, por meio de regressão multivariável de Poisson, com variância robusta. O teste deviance foi adotado para avaliar a qualidade do modelo ajustado. Todas as análises foram corrigidas pelo efeito do desenho, utilizando o comando complex samples do programa estatístico SPSS ${ }^{\circledR}$ versão 22.0.

O projeto desta pesquisa foi aprovado pelo Comitê de Ética em Pesquisa da Universidade Estadual de Montes Claros (Unimontes). Todos os preceitos éticos da Resolução 466/2012 foram devidamente respeitados.

\section{Resultados}

Seguindo os critérios de investigação do DUSI um total de 1.265 adolescentes, distribuídos em 21 escolas, foram investigados. A maioria tinha entre 16 e 17 anos, era do sexo feminino, se autodeclarou pardo, possuía renda familiar entre 1 e 2 salários mínimos, se autodeclararam de classe econômica $\mathrm{A}$ e $\mathrm{B}$, matriculados no $1^{\circ}$ ano e no turno matutino (Tabela 1).

A prevalência do consumo de bebidas alcoólicas pelos escolares foi $47,2 \%$ e mostrou associação com conflitos familiares, supervisão dos pais e qualidade do relacionamento, conforme descrito na Tabela 2. De acordo com as afirmações dos adolescentes, constatou-se que 69,9\% (121) deles tinham algum membro da família (mãe, pai, ir- 


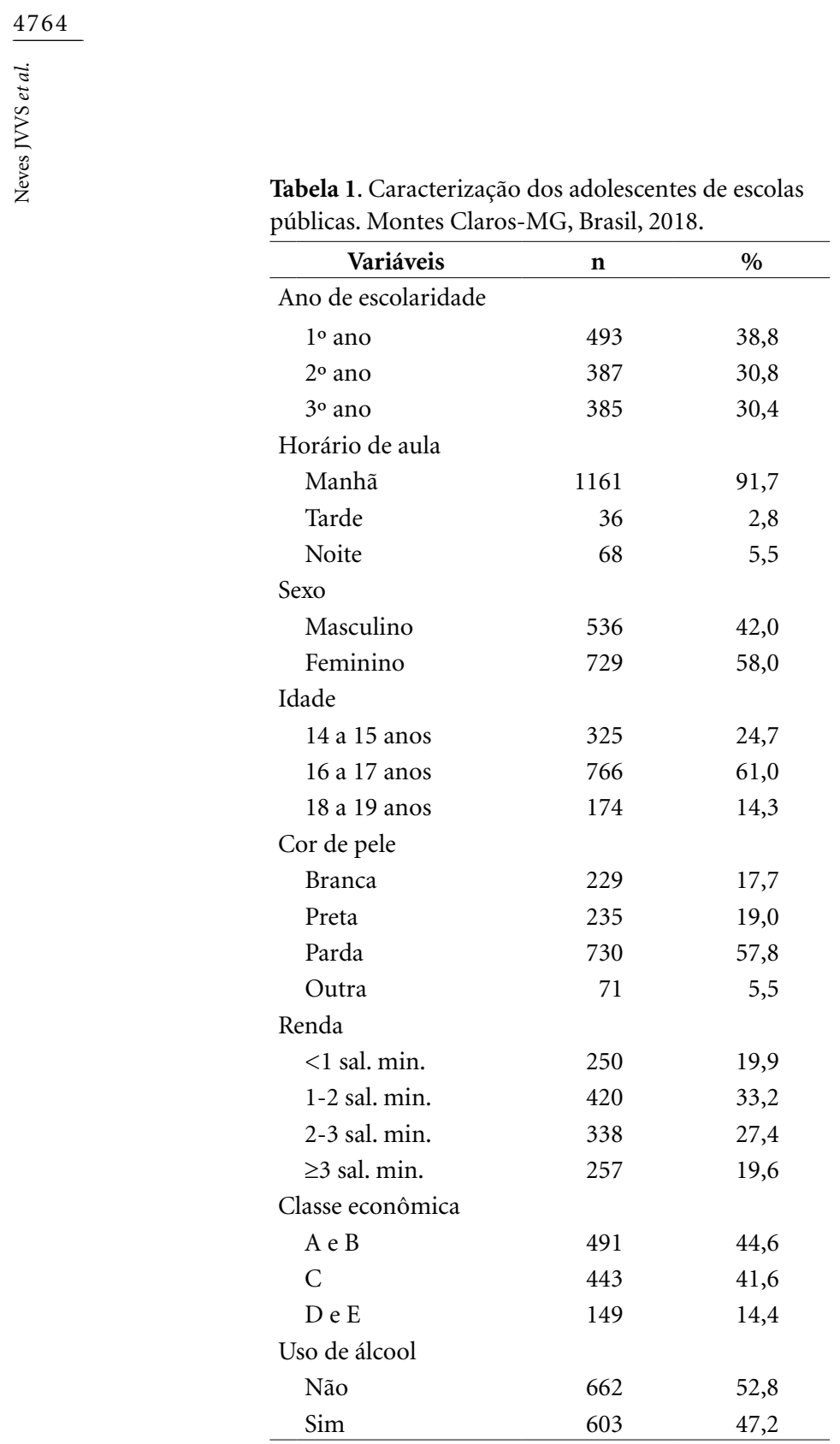

Fonte: Elaborado pelos autores.

mão ou irmã) que usou maconha ou cocaína no último ano; 56,7\% (230) tiveram discussões frequentes com seus pais ou responsáveis que envolveram gritos e berros; 49\% (305) relataram que seus pais ou responsáveis desconheciam o que realmente pensam ou sentem sobre as coisas que lhe são importantes; 50,8\% (262) confessaram que seus pais ou responsáveis estão fora de casa a maior parte do tempo; e 49,4 (161) se sentem infelizes em relação ao local em que vivem; 50,3\% (273) disseram que seus pais ou responsáveis desconhecem o que eles gostam e não gostam; $59,8 \%$ (118) afirmaram que sentem que seus pais ou responsáveis não se importam ou não cuidam deles. A Tabela 2 apresenta ainda a análise biva- riada utilizada para verificar a associação entre o uso de álcool e essas variáveis, ao nível de $\mathrm{p}<0,20$.

A razão de prevalência ajustada para uso de álcool e conflitos familiares está apresentada na Tabela 3. Ao nível de $\mathrm{p}<0,05$, as seguintes variáveis apresentaram associação estatisticamente significativa com o uso de álcool: "Algum membro de sua família (mãe, pai, irmão ou irmã) usou maconha ou cocaína no último ano?", "Você tem tido discussões frequentes com seus pais ou responsáveis que envolvam gritos e berros?" e "Seus pais ou responsáveis frequentemente desconhecem onde você está ou o que você está fazendo?”.

\section{Discussão}

O presente estudo verificou que cerca de 50\% dos estudantes consumiram bebidas alcoólicas no último mês, mostrando associação significativa com vínculo familiar e social conflituoso. Situações como ter algum membro da família que fez uso de maconha ou cocaína no último ano apresentou associação com o uso de álcool pelos adolescentes. O uso de drogas e bebidas alcoólicas por parte dos pais e comportamento antissocial são fatores de risco para o consumo de álcool e outras drogas nessa população ${ }^{13}$. $\mathrm{O}$ consumo de substâncias psicoativas é um comportamento social aprendido, a partir das interações primárias do adolescente com a família, escola e amigos ${ }^{14}$.

A família e a escola são instituições as quais, o adolescente costuma ter os seus primeiros contatos com as drogas, ambientes onde há um vínculo afetivo associado ${ }^{15}$. Os resultados deste estudo identificaram associação entre uso de álcool por algum membro familiar a ponto de causar problemas em casa, no trabalho ou com amigos e o uso pelos adolescentes. Achados em Horta et al. ${ }^{16}$ corroboram condições socioeconômicas e vivência de violência doméstica associadas ao uso de drogas pelos adolescentes.

Conforme achados deste estudo, Murray ${ }^{17}$ atesta que adolescentes filhos de pais que já foram encarcerados têm maior probabilidade de fazerem uso de drogas lícitas e ilícitas, apresentarem comportamentos sexualizados precocemente e passarem pelo sistema judiciário. Bucher ${ }^{18}$ afirma que os jovens que são marcados por diversas falhas na estruturação pessoal, e por perturbações familiares ou pessoais precocemente, caminham em direção à marginalização, apresentando maior chance de serem usuários de drogas.

De acordo com os resultados deste estudo, a frequência de discussões com pais ou respon- 
Tabela 2. Análise bivariada de uso de álcool e conflitos familiares em adolescentes de escolas públicas. Montes ClarosMG, Brasil, 2018.

\begin{tabular}{|c|c|c|c|c|}
\hline \multirow{2}{*}{ Conflitos familiares } & \multicolumn{4}{|c|}{ Uso de álcool } \\
\hline & Não (\%) & $\operatorname{Sim}(\%)$ & Total (\%) & p-valor \\
\hline \multicolumn{5}{|c|}{$\begin{array}{l}\text { 1. Algum membro de sua família (mãe, pai, irmão ou irmã) usou } \\
\text { maconha ou cocaína no último ano? }\end{array}$} \\
\hline $\mathrm{Não}^{*}$ & $610(56,3)$ & $482(43,7)$ & $1092(100,0)$ & \\
\hline Sim & $52(30,1)$ & $121(69,9)$ & $173(100,0)$ & $<0,001$ \\
\hline \multicolumn{5}{|c|}{$\begin{array}{l}\text { 2. Algum membro de sua família usou álcool a ponto de causar } \\
\text { problemas em casa, no trabalho ou com amigos? }\end{array}$} \\
\hline Não* & $515(55,2)$ & $423(44,8)$ & $938(100,0)$ & \\
\hline $\operatorname{Sim}$ & $147(46,0)$ & $180(54,0)$ & $327(100,0)$ & 0,019 \\
\hline \multicolumn{5}{|c|}{ 3. Algum membro de sua família foi preso no último ano? } \\
\hline $\mathrm{Não}^{*}$ & $581(54,6)$ & $494(45,4)$ & $1075(100,0)$ & \\
\hline $\operatorname{Sim}$ & $81(42,5)$ & $109(57,5)$ & $190(100,0)$ & 0,011 \\
\hline \multicolumn{5}{|c|}{$\begin{array}{l}\text { 4. Você tem tido discussões frequentes com seus pais ou responsáveis que } \\
\text { envolvam gritos e berros? }\end{array}$} \\
\hline Não* & $490(57,3)$ & $373(42,7)$ & $863(100,0)$ & \\
\hline $\operatorname{Sim}$ & $172(43,3)$ & $230(56,7)$ & $402(100,0)$ & $<0,001$ \\
\hline \multicolumn{5}{|l|}{ 5. Sua família dificilmente faz coisas juntas? } \\
\hline Não* & $381(54,0)$ & $328(46,0)$ & $709(100,0)$ & \\
\hline Sim & $281(51,3)$ & $275(48,7)$ & $556(100,0)$ & 0,477 \\
\hline \multicolumn{5}{|c|}{$\begin{array}{l}\text { 6. Seus pais ou responsáveis desconhecem o que você gosta e o que não } \\
\text { gosta? }\end{array}$} \\
\hline $\mathrm{Não}^{*}$ & $396(55,1)$ & $330(44,9)$ & $726(100,0)$ & \\
\hline Sim & $266(49,7)$ & $273(50,3)$ & $539(100,0)$ & 0,068 \\
\hline \multicolumn{5}{|c|}{ 7. Na sua casa falta regras claras sobre o que você pode e não pode fazer? } \\
\hline Não* & $543(53,5)$ & $481(46,5)$ & $1024(100,0)$ & \\
\hline Sim & $119(49,9)$ & $122(50,1)$ & $241(100,0)$ & 0,282 \\
\hline \multicolumn{5}{|c|}{$\begin{array}{l}\text { 8. Seus pais ou responsáveis desconhecem o que você realmente pensa ou } \\
\text { sente sobre as coisas que só importante para você? }\end{array}$} \\
\hline $\mathrm{Não}^{*}$ & $352(54,5)$ & $298(45,5)$ & $650(100,0)$ & \\
\hline Sim & $310(51,0)$ & $305(49,0)$ & $615(100,0)$ & 0,035 \\
\hline \multicolumn{5}{|l|}{ 9. Seus pais ou responsáveis brigam muito entre si? } \\
\hline $\mathrm{Não}^{*}$ & $517(52,6)$ & $470(47,4)$ & $987(100,0)$ & \\
\hline $\operatorname{Sim}$ & $145(53,5)$ & $133(46,5)$ & $278(100,0)$ & 0,761 \\
\hline \multicolumn{5}{|c|}{$\begin{array}{l}\text { 10. Seus pais ou responsáveis frequentemente desconhecem onde você } \\
\text { está ou o que você está fazendo? }\end{array}$} \\
\hline $\mathrm{Não}^{*}$ & $567(57,6)$ & $424(42,4)$ & $991(100,0)$ & \\
\hline Sim & $95(35,9)$ & $179(64,1)$ & $274(100,0)$ & $<0,001$ \\
\hline \multicolumn{5}{|c|}{ 11. Seus pais ou responsáveis estão fora de casa a maior parte do tempo? } \\
\hline $\mathrm{Não}^{*}$ & $420(55,2)$ & $341(44,8)$ & $761(100,0)$ & \\
\hline Sim & $242(49,2)$ & $262(50,8)$ & $504(100,0)$ & 0,068 \\
\hline \multicolumn{5}{|c|}{$\begin{array}{l}\text { 12. Você sente que seus pais ou responsáveis não se importam ou não } \\
\text { cuidam de você? }\end{array}$} \\
\hline $\mathrm{Não}^{*}$ & $585(55,1)$ & $485(44,9)$ & $1070(100,0)$ & \\
\hline Sim & $77(40,2)$ & $118(59,8)$ & $195(100,0)$ & 0,001 \\
\hline \multicolumn{5}{|c|}{ 13. Você se sente infeliz em relação ao local no qual você vive? } \\
\hline $\mathrm{Não}^{*}$ & $505(53,6)$ & $442(46,4)$ & $947(100,0)$ & \\
\hline $\operatorname{Sim}$ & $157(50,6)$ & $161(49,4)$ & $318(100,0)$ & 0,375 \\
\hline \multicolumn{5}{|l|}{ 14. Você se sente em perigo em casa? } \\
\hline $\mathrm{Não}^{\star}$ & $627(53,2)$ & $563(46,8)$ & $1190(100,0)$ & \\
\hline Sim & $35(47,4)$ & $40(52,6)$ & $75(100,0)$ & 0,386 \\
\hline
\end{tabular}

Fonte: Elaborado pelos autores. 
Tabela 3. Razão de prevalência (RP) ajustada para Uso de Álcool e Conflitos familiares em adolescentes de escolas públicas. Montes Claros-MG, Brasil, 2018.

\begin{tabular}{|c|c|c|c|}
\hline Conflitos familiares & $\mathbf{R P}$ & IC 95\% & p-valor \\
\hline \multicolumn{4}{|c|}{$\begin{array}{l}\text { 1. Algum membro de sua família (mãe, pai, irmão ou irmã) usou maconha } \\
\text { ou cocaína no último ano? }\end{array}$} \\
\hline Não* & 1 & - & \\
\hline Sim & 1,46 & $1,29-1,65$ & $<0,001$ \\
\hline \multicolumn{4}{|c|}{$\begin{array}{l}\text { 4. Você tem tido discussões frequentes com seus pais ou responsáveis que } \\
\text { envolvam gritos e berros? }\end{array}$} \\
\hline Não* & 1 & - & \\
\hline Sim & 1,19 & $1,05-1,34$ & 0,005 \\
\hline \multicolumn{4}{|c|}{$\begin{array}{l}\text { 10. Seus pais ou responsáveis frequentemente desconhecem onde você está } \\
\text { ou o que você está fazendo? }\end{array}$} \\
\hline Não* & 1 & - & \\
\hline Sim & 1,38 & $1,22-1,56$ & $<0,001$ \\
\hline
\end{tabular}

Fonte: Elaborado pelos autores.

sáveis envolvendo gritos e berros foi estatisticamente associado ao uso de álcool. Porém não foi encontrada associação em briga entre pais ou responsáveis e o uso dessa substância. As relações familiares baseadas em violência aumentam a possibilidade dos adolescentes consumirem substâncias alucinógenas e pode-se compreender como uma consequência da violência transpassada no seio familiar ${ }^{19}$. O âmbito familiar, local dos primeiros contatos do sujeito com o mundo social está relacionado, a partir de outros estu$\operatorname{dos}^{16,20}$, a um fator de proteção dos adolescentes quanto às drogas, tais como morar com os pais e supervisão familiar. Entretanto, o desequilíbrio na relação familiar, sobretudo contextos de violência, tem potencial de comprometimento da saúde dos adolescentes. O uso de álcool e outras drogas está intimamente relacionado com tentativa de fuga de uma vivência social desfavorável, em especial nas violentas relações familiares ${ }^{16,21}$.

No que se refere à realização de atividades em conjunto com a família, os pesquisados relataram dificilmente realizá-las e fizeram uso de álcool, entretanto nesta pesquisa não foi encontrada associação. Porém, mais da metade dos adolescentes disseram que seus pais ou responsáveis desconhecem o que esses gostam e não gostam. Malta et al. ${ }^{22}$ ressaltam que os adolescentes cujo os pais não sabem o que esses fazem, não alimentam juntos e não se importam se os filhos bebem, foram fatores associados a maior chance deconsumo de álcool. O desconhecimento dos pais sobre o que os adolescentes fazem e como agem foram fatores de risco para o maior consumo dessa substância ${ }^{11,22}$.
Em relação ao conhecimento dos pais ou responsáveis sobre o que o adolescente realmente pensa ou sente sobre as coisas que lhe são importantes, este estudo verificou alto percentual de desconhecimento e associação com uso de álcool. Para o adolescente, é importante perceber o interesse dos pais por suas atividades e sua vida de um modo geral. A preocupação em dividir momentos e compartilhar atividades são elementos que podem levar à manutenção de um bom vínculo e interação com os pais ${ }^{5,11}$, ao contrário, a falta dessa relação pode acarretar distanciamento dos filhos do ambiente familiar e prejudicar a integralidade da saúde ${ }^{5}$. Os laços familiares e o bom relacionamento com e entre os pais foram fatores de proteção quanto ao uso de álcool e outras drogas ${ }^{3,11}$.

Constatou-se que os pais de mais de $50 \%$ dos pesquisados estão fora de casa a maior parte do tempo. Ao analisar se os pais ou responsáveis desconhecem onde o adolescente está ou o que está fazendo, verificou-se associação significativa com o uso de álcool entre $64,1 \%$ dos pesquisados. $\mathrm{O}$ desequilíbrio na relação familiar, em especial, a negligência parental tem potencial de comprometimento da saúde integral dos adolescentes ${ }^{13}$. Dificuldades nas interações dos adolescentes com a família em especial, são potenciais fatores de risco a desenvolvimento de complicações na vida do sujeito ${ }^{14}$.

Ao relatarem sobre a presença de regras claras sobre o que podem e não podem fazer, mais de 50\% dos adolescentes afirmaram faltar regras e consumirem álcool, entretanto neste item não se observou associação, contrariando os achados 
de outro estudo ${ }^{23}$ que ratifica a presença de forte relação entre o uso de álcool por adolescentes e a supervisão familiar. Regras, disciplina e a presença dos pais têm favorecido o desenvolvimento de competências sociais e a adesão a comportamentos protetores frente ao álcool, como a seleção dos amigos e reconhecimento dos riscos envolvidos ${ }^{24}$. Segundo $\mathrm{Bee}^{25}$ a família é a instituição responsável pelo processo de socialização e assim, adquirem-se comportamentos e valores apropriados ao meio social. Este mesmo autor afirma que a internalização das normas e regras familiares proporcionam um desempenho social melhor, além de possibilitar maior aquisição de autonomia.

Observou-se ainda que aproximadamente $60 \%$ dos adolescentes consumiram álcool e relataram sentir que seus pais não se importam ou não os cuidam. A proximidade e o diálogo com os pais possibilita ao adolescente se expressar e expor suas ideias, além de garantir maior manifestação de afeto e segurança familiar, no entanto, atualmente pais e filhos tendem a interagir menos ${ }^{5}$. Há diversos prejuízos advindos do uso de drogas pelos adolescentes, no que tange a comportamentos de riscos, atraso no desenvolvimento, obtenção de autocontrole e autoestima ${ }^{26}$.

Sobre o sentimento em relação ao local no qual vivem e se sentiam em perigo estando em casa, não houve associação com o uso de álcool, dados que se opõem aos achados anteriormente discutidos. Constatou-se ainda incoerência nos relatos quanto aos itens "Renda" e "Classe Econômica”, 33,2\% afirmaram ter renda média familiar entre 1 e 2 dois salários mínimos e pertencerem a classe econômica A e B $(44,6 \%)$. Tal incoerência pode-se justificar pela possibilidade de um viés consequente do autorrelato dos estudantes sobre um tema no qual eles não possuíam conhecimento. No entanto, a desigualdade social e a pobreza contribuem efetivamente para a deterioração das relações afetivas e familiares, engendrando situações estressantes e danos a diversas esferas da vida do sujeito ${ }^{27}$. A relação familiar disfuncional é constitutiva da experiência depressiva no público adolescente ${ }^{9}$, assim como o uso de álcool pelos adolescentes está fortemente relacionada a dificuldades psicológicas, entre outras ${ }^{28}$. Nesse sentido, o desenvolvimento social, cognitivo e emocional do sujeito é afetado negativamente pelo uso de álcool.

A perda de questionários considerados inválidos após aplicação dos parâmetros da "Escala de Mentiras” pode indicar uma limitação deste estudo, no entanto, a amostra final foi significativa. Ademais, a aplicaçãodesses parâmetros permitiu maior confiabilidade dos resultados.

Conclui-se que a adolescência é uma fase complexa do desenvolvimento humano que envolve as dimensões biológica, psicológica e social. A alta taxa de adolescentes escolares que consomem álcool encontrada neste estudo é preocupante, passível de complicações da saúde na sua integralidade, sendo fundamental investimentos para uma relação familiar harmonizada e o fortalecimento dos laços afetivo-familiares, a fim de se estabelecer um vínculo protetivo. A implementação de programas de conscientização sobre os riscos consequentes ao uso de álcool durante a adolescência pode favorecer o acompanhamento dos adolescentes pelas instituições familiar, escolar e social. A articulação de intervenções entre escola e Estratégia de Saúde da Família (ESF) podem contribuir para o enriquecimento dos vínculos familiares e a saúde mental dos envolvidos. Nesse sentido, o investimento nas políticas públicas e o incremento da rede de proteção à saúde tornam-se necessárias para garantir os direitos dos adolescentes, transmitir valores e normas sociais e prevenir o uso de álcool.

\section{Colaboradores}

RRV Silva participou da concepção e desenho do estudo e da análise dos dados. MTC Almeida, JVVS Neves, LA Carvalho e MA Carvalho participaram da análise e interpretação dos dados, da redação e revisão crítica do manuscrito. ETC Silva e MLTS Alves contribuíram na redação do manuscrito. MF Silveira colaborou na análise e interpretação dos dados. RRV Silva e MF Silveira trabalharam na revisão final do manuscrito. 


\section{Referências}

1. World Health Organization (WHO). Young People's Health: a Challenge for Society. Report of a WHO Study Group on Young People and Health for All. Technical Report Series 731. Genebra: WHO; 1986.

2. Teixeira PS, Stefanini MCB, Martins RA, Cruz LAN. Desenvolvimento cognitivo e sintomas depressivos em adolescentes que fazem uso de bebidas alcoólicas. Rev Eletr Saude Mental Alcool Drogas 2011; 7(1):3-9.

3. Giacomozzi AI, Itokasu MC, Luzardo AR, Figueiredo CDS, Vieira M. Levantamento sobre uso de álcool e outras drogas e vulnerabilidades relacionadas de estudantes de escolas públicas participantes do programa Saúde do Escolar/saúde e prevenção nas escolas no município de Florianópolis. Rev Saude Soc 2012; 21(3):612-622.

4. Valle LELR, Mattos MJVM. Adolescência: as contradições da idade. $2^{\text {a }}$ ed. Rio de Janeiro: Wak Editora; 2010.

5. Pratta EMM, Santos MA. Família e Adolescência: a influência do contexto familiar no desenvolvimento psicológico de seus membros. Rev Psicol Estud 2007; 12(2):247-256.

6. Melo AKS, Moreira V. Fenomenologia da queixa depressiva em adolescentes: um estudo crítico-cultural. Rev Aletheia 2008; 27(1):51-64.

7. Mendonça AKRH, Jesus CVF, Lima SO. Fatores associados ao consumo alcoólico de risco entre universitários da área da saúde. Rev Bras Educ Med 2018; 42(1):205-213.

8. Brasil. Ministério do Planejamento, Orçamento e Gestão. Ministério da Saúde (MS). Pesquisa Nacional de Saúde do Escolar: 2012. Rio de Janeiro: IBGE; 2013.

9. Bock AMB, Furtado O, Teixeira MLT. Psicologias: Uma introdução ao estudo de Psicologia. 14a ed. São Paulo: Saraiva; 2008.

10. Wallander JL, Schimitt M, Koot HM. Quality of life measurement in children and adolescents: issues, instruments, and applications. J Clin Psychol 2001; 57(4):571-585.

11. Oliva A. Desenvolvimento da personalidade durante a adolescência. In: Coll C, Palácios J, Marchesi A. Desenvolvimento psicológico e educação: psicologia evolutiva. 2a ed. Porto Alegre: Artes Médicas; 2007. p. 335-349.

12. Fidalgo TM, Tarter R, Silveira ED, Kirisci L, Silveira DX. Validation of a short version of the revised drug use screening inventory in a Brazilian sample of adolescents. Am J Addict 2010; 19(4):364-367.

13. Andrade SSCA, Yokota RTC, Sá NNB, Silva MMA, Araújo WN, Mascarenhas MDM, Malta DC. Relação entre violência física, consumo de álcool e outras drogas e bullying entre adolescentes escolares brasileiros. Cad Saude Publica 2012; 28(9):1725-1736.

14. Schenker M, Minayo MCS. Fatores de risco e de proteção para o uso de drogas na adolescência. Cien Saude Colet 2005; 10(3):2879-2890.

15. Faggiano F, Vigna-Taglianti FD, Versino E, Zambon A, Borraccino A, Lemma P. School-based prevention for illicit drugs use: a systematic review. Prev Med 2008; 46(5):385-396.

16. Horta RL, Horta BL, Costa AWN, Prado RR, Oliveira-Campos M, Malta DC. Uso na vida de substâncias ilícitas e fatores associados entre escolares brasileiros, Pesquisa Nacional de Saúde do Escolar (PeNSE, 2012). Rev Bras Epidemiol 2014; (Supl. PeNSE):31-45.
17. Murray J. The cycle of punishment: Social exclusion of prisoners and their children. Criminol Criminal Justice 2007; 7(1):55-81.

18. Bucher R. Drogas e drogadição no Brasil. Porto Alegre: Artes Médicas; 1992.

19. Mota RS, Gomes NP, Estrela FM, Silva MA, Santana JD, Campos LM, Cordeiro KCC. Prevalência e fatores associados à vivência de violência intrafamiliar por adolescentes escolares. Rev Bras Enferm 2018; 71(3):1086-1091.

20. Malta DC, Machado IE, Felisbino-Mendes MS, Prado RR, Pinto AMS, Oliveira-Campos M, Souza MFM, Assunção AA. Uso de substâncias psicoativas em adolescentes brasileiros e fatores associados: Pesquisa Nacional de Saúde dos Escolares, 2015. Rev Bras Epidemiol 2018; 21(Supl. 1):E180004.supl.1.

21. Davies PJ, Dreyer YA. Pastoral psychological approach to domestic violence in South Africa. HTS Teologiese Study 2014; 70(3):1-8.

22. Malta DC, Mascarenhas MDM, Porto DL, Barreto SM, Morais Neto OL. Exposição ao álcool entre escolares e fatores associados. Rev Saude Publica 2014; 48(1):52-62.

23. Machado IE, Felisbino-Mendes MS, Malta DC, Velasquez-Melendez G, Freitas MIF, Andreazzi MAR. Supervisão dos pais e o consumo de álcool por adolescentes brasileiros: análise dos dados da Pesquisa Nacional de Saúde do Escolar de 2015. Rev Bras Epidemiol 2018; 21(Supl. 1):e180005.

24. Wang C, Hipp JR, Butts CT, Jose R, Lakon CM. Alcohol use among adolescent youth: the role of friendship networks and family factors in multiple school studies. PLoS One 2015; 10(3):e0119965.

25. Bee HL. The developing child. 7a ed. New York: HarperCollins College Publishers; 1995.

26. Scivolleto S, Morihisa RS. Conceitos básicos em dependência de álcool e outras drogas na adolescência. $J$ Bras Depend Quim 2001; 2(1):30-33.

27. Ramires VRR, Falcke D. Fatores de risco e proteção para vínculos familiares no sul do Brasil. Rev Psicol Teoria Prática 2018; 20(1):126-140.

28. Aires S, Neves S, Cálix MJ, Figueiredo C, Silveira A. Alcoolismo na adolescência: a realidade de um Serviço de Pediatria. Rev Pediatr Centro Hosp Porto 2014; 23(1):8-11.

Artigo apresentado em 11/03/2020

Aprovado em 24/08/2020

Versão final apresentada em 26/08/2020

Editores-chefes: Romeu Gomes, Antônio Augusto Moura da Silva 